\title{
Localisation du récepteur des androgènes dans le testicule
}

\author{
C.A. SUAREZ-QUIAN*, B.O. OKE**, W. VorNBERGER (TRADUCTION H. GÉRARD) \\ *Department of cell Biology Georgetown Medical center, 3900 Reservoir Road, N.Y. Washington, \\ D.C. 20007 U.S.A. ; **Department of Veterinary Anatomy, University of Ibadan, Nigeria
}

\section{RESUME}

La question de savoir si les cellules de la lignée germinale possèdent ou non des récepteurs aux androgènes reste débattue. Dans ce travail nous avons déterminé la distribution des récepteurs aux androgènes dans le testicule de rat et de souris à l'aide d'un anticorps polyclonal de lapin purifié par affinité, dirigé contre un peptide de 21 acides aminés de la région terminale du récepteur des androgènes $(R A)$, marqué par le système biotine-streptavidine immunoperoxidase.

La spécificité de l'anticorps est attestée par les points suivants :

1) Présence d'une bande spécifique approximativement à $110 \mathrm{kD}$ en Western immunoblot ; 2) Elimination spécifique du marquage par pré-adsorption de l'anticorps avec le peptide de 21 acides aminés ; 3) Mise en évidence d'une relation entre l'intensité du marquage dans toutes les cellules RA positives en fonction de la dilution des antisérums ; 4) Mise en évidence dans tous les tissus connus pour exprimer de façon importante des RA (par exemple l'épididyme, la prostate, les vésicules séminales) d'un marquage extrêmement intense au niveau du noyau des cellules épithéliales ; 5)
Obtention d'un réaction RA positive avec les anticorps dans les lignées de cellules prostatiques connues pour exprimer les récepteurs aux androgènes ; 6) Positivation de cellules COS-7 après leur transfection par un vecteur exprimant le récepteur aux androgènes de rat. De plus la distribution intracellulaire des RA dans les cellules COS-7 est fonction de la présence d'androgènes dans le milieu.

L'immunolocalisation des récepteurs aux androgènes montre les points suivants: à l'intérieur du compartiment interstitiel chez le rat adulte des RA sont détectés dans quelques cellules de Leydig et dans toutes les cellules musculaires lisses formant la paroi des vaisseaux sanguins, alors que les cellules endothéliales restent négatives. Dans les tubes séminifères les RA sont observés dans tous les noyaux des cellules myoïdes péritubulaires, mais pas dans les couches plus périphériques correspondant aux cellules endothéliales des vaisseaux lymphatiques. Dans les cellules de Sertoli le marquage nucléaire des RA est spécifique du stade de l'épithélium séminifère : la positivité du marquage commence à devenir évidente vers le stade IV tardif du cycle, atteint un pic très fort aux stades VII et VIII puis disparaît com- 
plètement. Un immunomarquage spécifique a également été mis en évidence dans les noyaux des spermatides allongées stade $\mathrm{Xl}$, stade auquel l'élongation nucléaire est déjà apparente mais sans que la condensation de la chromatine soit déjà commencée. Dès le début de la condensation de la chromatine des spermatides allongées, l'immunomarquage nucléaire disparaît en même temps que le cytoplasme devient positif. Globalement les mêmes observations ont été faites sur le testicule de Souris adulte, excepté le fait que, dans cette espèce, toutes les cellules de Leydig sont très fortement RA positives.

Afin de confirmer l'immunomarquage des RA nous avons réalisé des études d'hybridation in situ dans le testicule de Rat adulte à l'aide d'une sonde antisens ARNc de 236 paires de bases (I'ADNc de RA nous a été donné par le Docteur Chang, Université du Wisconsin, Madison, WI). Un important signal d'hybridation a été observé à la base de l'épithélium séminifère, dans la région occupée par les cellules de Sertoli et les spermatogonies. Ceci nous a conduit a réexaminer les résultats de l'immunomarquage afin de déterminer si les spermatogonies étaient également positives pour les anticorps anti RA. Nos résultats préliminaires sont en faveur de la présence de RA dans certaines populations spermatogoniales. Au total, ces résultats concordent avec des observations antérieures suggérant que les RA sont présents dans les cellules somatiques du testicule; de ce fait, ce sont ces types cellulaires qui vraisemblablement répondent aux androgènes circulants pour contrôler la spermatogenèse. Cependant nos résultats montrent également un immunomarquage de certains éléments de la lignée germinale ce qui réactualise la controverse concernant le fait de savoir si la lignée germinale peut répondre ou non directement aux androgènes.

Mots clés : Androgènes, cellules germinales, immunohistochimie, récepteurs des androgènes, testicule.

\section{INTRODUCTION}

Le site d'action des androgènes au niveau du testicule a été un sujet de controverse depuis de nombreuses années. Initialement, sur la base d'étude de liaison avec des molécules marquées, les résultats semblaient en faveur de l'hypothèse que les androgènes pourraient agir directement à la fois sur les cellules somatiques et sur les cellules de la lignée germinale; cependant un certain nombre de considérations théoriques avaient conduit Fritz à publier une revue critique par laquelle il a suggéré que les androgènes agissaient seulement au niveau des cellules somatiques [1].Plus récemment, la production d' anticorps contre les récepteurs des androgènes (RA), et leur utilisation pour des études d'immunolocalisation par plusieurs laboratoires avaient conduit à espérer que cette controverse allait se trouver définitivement éclaircie [2-4]. Malheureusement, des résultats contradictoires obtenus par différents investigateurs, y compris notre groupe, ont contribué à faire renaître le débat sur l'existence de RA dans certaines cellules de la lignée germinale. L'existence de tels récepteurs impliquerait la possiblité très "excitante" que certaines cellules de la lignée germinale puissent répondre directement à la testostérone. Dans cet article nous apportons des résultats immunohistochimiques qui nous conduisent à conclure que certaines cellules de la lignée germinale expriment des récepteurs aux androgènes.

\section{MATERIEL ET METHODES}

\section{Anticorps anti RA}

L'anticorps PG21 est un anticorps polyclonal de lapin purifié par affinité dirigé 
contre un peptide de synthèse correspondant au 21 premiers acides aminés du RA de rat. Sa validité pour l'immunodétection des récepteurs aux androgènes de rat a été établie antérieurement [5].

\section{Immunocytochimie}

Toutes les techniques utilisées sont réalisées exactement comme cela a déjà été décrit par ailleurs en détail pour des testicules de rat et de souris inclus à la paraffine [6]. Cependant, avant l'immunomarquage, mais après la réhydratation des coupes, celles-ci sont placées dans un tampon $0.1 \mathrm{M}$ de citrate, $\mathrm{pH} 6.0$ et traitées pendant 30 minutes dans un four à micro-ondes à 600 $W$. On prend soin que le tampon n'arrive pas à ébulition ce qui entraînerait le déssèchement des coupes. Après le passage au four à micro-ondes, les coupes sont ramenées à température ambiante et sont soumises à la procédure d'immunocoloration (biotine- streptavidine immunoperoxidase). Dans ce protocole la réaction positive est caractérisée par la formation d'un précipité rouge brun au site de l'interaction antigène-anticorps. Après la réaction immune, le tissu est contrecoloré à l'hématoxyline qui colore de façon générale les coupes en bleu, en particulier les noyaux. Malheureusement, du fait que les RA sont des protéines nucléaires à l'équilibre, la contrecoloration par l'hématoxyline dans les délais habituellement utilisés à tendance à masquer la couleur rouge brun des noyaux des cellules positives. Afin d'éviter ce problème, nos coupes n'ont été contrecolorées que pendant 2 à 3 secondes; ceci suffit cependant pour transformer la couleur rouge brun du précipité en une teinte plus pourpre. C'est cette teinte pourpre dans les noyaux qui a été considérée comme une réaction positive dans ce travail.

L'anticorps primaire contre les RA a été utilisé à une concentration allant de 2 microgrammes/millilitre à 23 microgrammes/millilitre. Les contrôles ont com- porté la préadsorption de l'anticorps primaire avec le peptide antigène en concentration dix fois excédentaire ou avec un peptide sans relation avec les RA avant son utilisation comme anticorps primaire.

L'immunomarquage obtenu avec l'anticorps préadsorbé a toujours été comparé aux résultats obtenus avec l'anticorps non préadsorbé sur des coupes sériées. De plus nous avons comparé les résultats obtenus par immunofluorescence parallèlement à la technique de coloration par biotine-streptadivine immunoperoxidase. La technique utilisée est identique à celle décrite ci-dessus si ce n'est que le deuxième anticorps a été remplacé par un anticorps de brebis anti lapin conjugué à la rhodamine (Cappel) à la dilution de 1/80. D'autres contrôles ont été réalisés comme il est décrit plus loin au "paragraphe résultats".

\section{RESULTATS}

\section{Localisation des RA}

Résultats généraux : L'observation des coupes de testicules de Rat adulte après immunomarquage montre une réaction positive à la fois dans le compartiment interstitiel et dans l'épithélium séminifere ainsi que dans la paroi des tubes (Figure 1). Dans le compartiment interstitiel le marquage spécifique des RA se trouve au niveau des cellules de Leydig et dans les cellules musculaires lisses entourant les vaisseaux. Chez le rat l'intensité du marquage varie d'une cellule à l'autre, même lorsqu'il s'agit de deux cellules adjacentes ; alors que certaines cellules de Leydig montrent une réaction très importante, d'autres sont à peine marquées, d'autres enfin se montrent tout à fait négatives (Figure 2A). Chez la Souris au contraire le marquage des cellules de Leydig est homogène, intense et il est présent dans toutes les cellules (Figure 2B). De plus, alors que dans les testicules de Rat les cellules de Leydig marquées le plus fortement atteignent à peine 


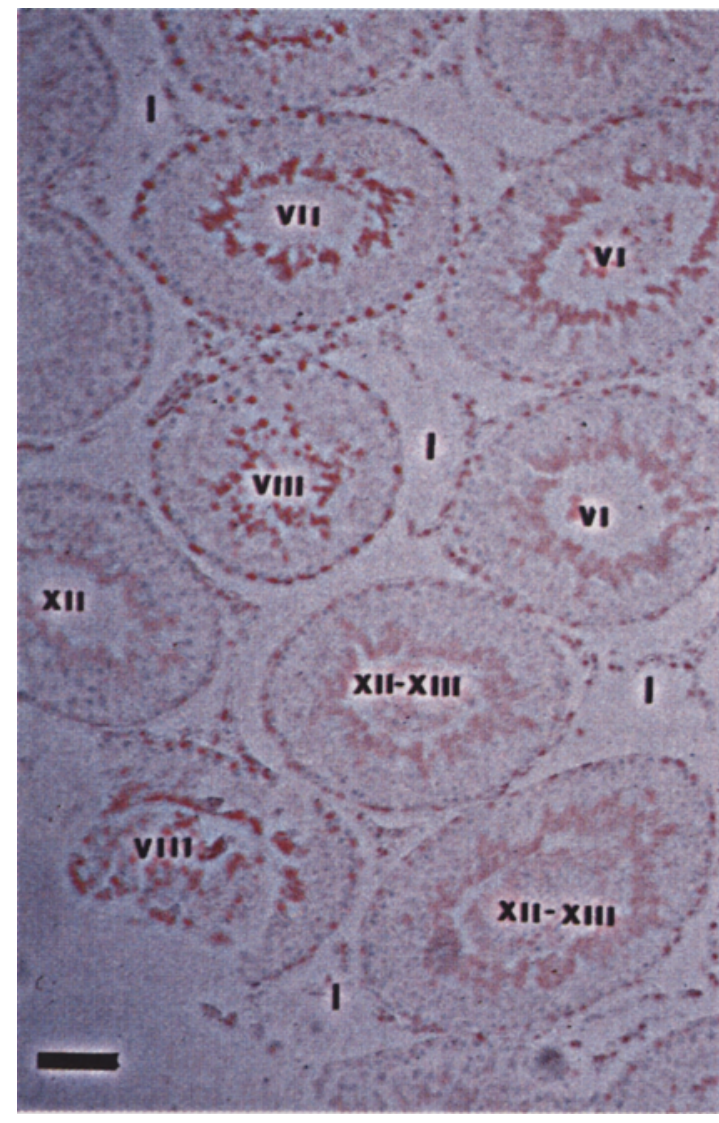

Figure 1 : Distribution des récepteurs des androgènes (AR) dans le testicule de rat adulte. Les tubes séminifères en coupes transversales montrent différents types d'associations cellulaires caractéristiques des stades de l'épithélium séminifère qui sont mentionnés en chiffre romain. On voit des noyaux des cellules de Sertoli localisés à la base de l'épithélium qui sont positifs pour les récepteurs aux androgènes pendant les stades moyens (VII-VIII), mais qui deviennent négatifs dans les stades plus avancés (IX-XIV). Le cytoplasme des spermatides allongées est également positif et l'intensité du marquage devient plus importante lorsque le cytoplasme forme les corps résiduels au stade VIII avant la spermiation. Au niveau de l'interstitium (I) on peut voir un marquage léger des cellules de Leydig. Barre d'échelle : $100 \mu \mathrm{m}$.
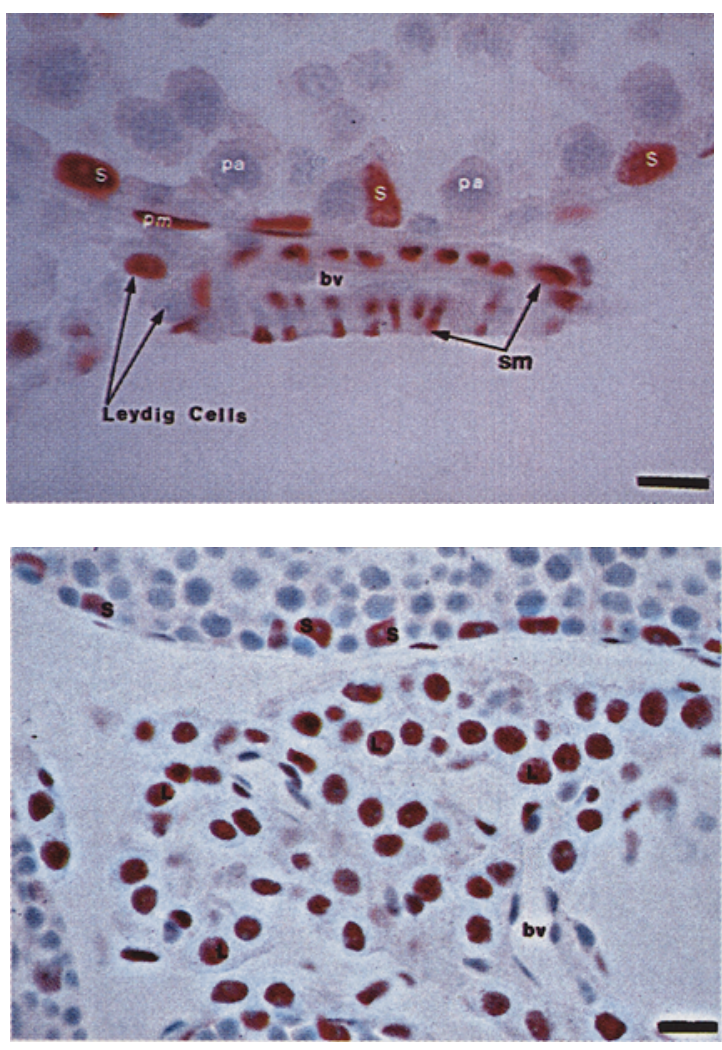

Figure 2 : Distribution des récepteurs aux androgènes dans l'interstitium chez le rat et la souris.

(A) Le principal marquage pour les récepteurs aux androgènes trouvés dans l'interstitium de rat est constitué par les noyaux des cellules musculaires lisses (sm) constituant la paroi des vaisseaux sanguins (bv). Les cellules de Leydig, marquées par des flèches, peuvent être soit positives soit complètement négatives. Les cellules de Sertoli (S) et les cellules péritubulaires myoüdes (pm) ont des noyaux positifs. Echelle : 10 $\mu m$.

(B) Dans l'interstitium, chez la souris, les cellules de Leydig (L) sont fortement marquées. L'intensité du marquage est similaire sinon plus forte que celle trouvée dans les noyaux des cellules de Sertoli (S). (bv)= vaisseaux sanguins.Barre d'échelle : $10 \mu \mathrm{m}$ 
l'intensité du marquage obtenu pour d'autres cellules somatiques, comme par exemple les cellules de Sertoli ou les cellules myoïdes péritubulaires, chez la Souris les cellules de Leydig présentent un marquage dont l'intensité est toujours au moins aussi grande que celles des autres cellules positives (Figure 2B). Enfin, aussi bien chez la souris que chez le rat, le marquage des noyaux des cellules musculaires lisses entourant les vaisseaux sanguins est en général assez intense alors que les cellules endothéliales bordant la lumière vasculaire restent négatives.

Au niveau du compartiment tubulaire la localisation des RA se fait selon deux grandes modalités générales à savoir : Stade dépendant ou constant (Figure 1).

Tout d'abord à l'intérieur de l'épithélium séminifere le cytoplasme des spermatides allongées est positif et l'intensité des marquages varie selon le stade du cycle de l'épithélium séminifêre. On voit en effet l'intensité du marquage cytoplasmique augmenter progressivement du stade XII au stade VIII. Après la spermiation, au stade VIII, le marquage présent dans les corps résiduels disparaît rapidement. Deuxièmement, les noyaux des cellules de Sertoli sont positifs et ce marquage est également stade spécifique. Dans les premiers stades du cycle de l'épithélium séminifère le marquage des noyaux des cellules de Sertoli est faible alors qu'il augmente progressivement dans les stades suivants pour atteindre un maximum au stade VIII (Figure 3A-C). Du stade IX au stade XIV, on observe aucun marquage dans les noyaux des cellules de Sertoli.

A l'inverse dans les noyaux des cellules myoïdes péritubulaires nous n'avons observé aucune différence dans l'intensité du marquage en fonction du cycle de l'épithélium séminifêre. De manière très caractéristique tous les noyaux de ces cellules myoïdes péritubulaires sont marqués de façon extrêmement intense, y compris du stade IX au stade XIV, période pendant laquelle les noyaux des cellules de Sertoli sont négatifs. Nous avons obtenu les mêmes résultats chez la Souris et chez le Degu, une souris saisonnière du Chili (résultats non montrés).

Un examen plus attentif du marquage des cellules de Sertoli au stade VII-VIII montre que leurs noyaux sont disposés dans deux orientations : leur grand axe est soit perpendiculaire à la paroi du tube, soit parallèle à cette paroi. Ces résultats sont en accord avec des observations antérieures sur l'orientation des noyaux des cellules de Sertoli.

Il convient de remarquer que certains des noyaux positifs détectés à la base de l'épithélium du tube séminifere ont une disposition similaire à celle des cellules positives pour c-kit, un marqueur spécifique des spermatogonies. Ceci nous a conduit à réaliser un double marquage immunocytochimique: pour c-kit d'une part et pour les RA d'autre part. Cependant, pour des raisons techniques (superposition des colorations), les résultats que nous avons obtenus jusqu'à présent ne nous permettent pas d'affirmer avec certitude que des cellules étaient positives pour les deux détections. Néanmoins le nombre de noyaux positifs pour les RA, détectés à la base des tubes séminifêres, est trop élevé pour pouvoir représenter uniquement des éléments sertoliens; nous proposons donc qu'une partie de ces noyaux représente une population de spermatogonies. Les résultas obtenus par hybridation in situ avec une sonde cRNA pour les RA du Rat sont également en faveur de cette interprétation (résultats non montrés).

Par ailleurs la positivité des spermatides allongées aux anticorps anti-RA a été examinée en détail afin de déterminer à quel stade la différenciation des récepteurs apparaissait. Les spermatides allongées de stade 11, stade auquel l'élongation nucléaire devient évidente mais sans qu'il y ait encore une condensation importante du 


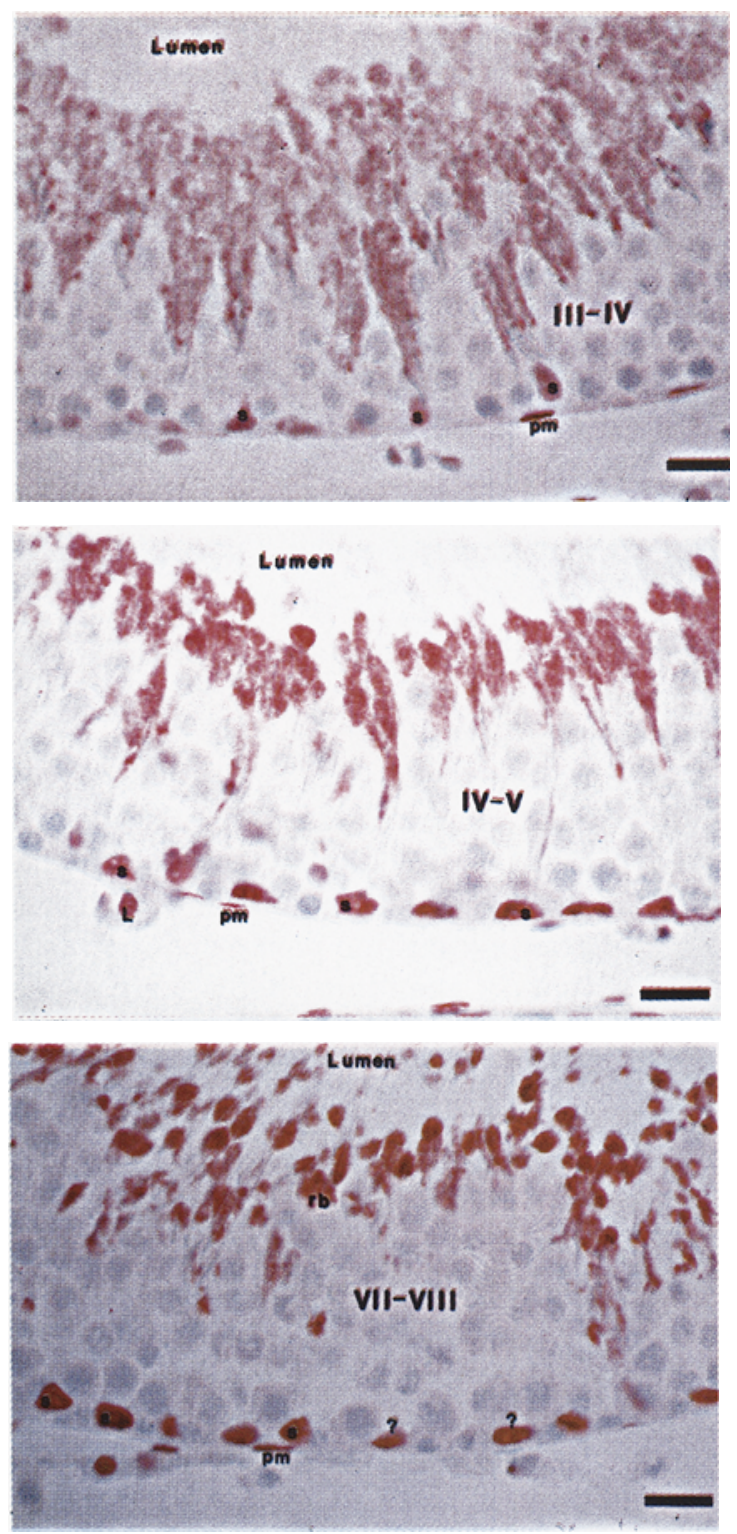

noyau sont positives au niveau de leur noyau (Figure 4). Dès que la chromatine commence à se condenser, au stade suivant, le marquage nucléaire des spermatides allongées disparait progressivement dans le sens cranibcaudal en même temps qu'un marquage cytoplasmique apparaît progressivement (résultats non montrés).

\section{Contrôles utilisés}

La validité des résultats d'une étude immunocytochimique repose essentiellement sur
Figure 3. Récepteurs aux androgènes dans les tubes séminifêres chez le rat.

(A) Pendant les premiers stades du cycle (III-IV) les noyaux des cellules de Sertoli (S) sont positifs pour les anticorps anti-récepteurs aux androgènes, mais restent marqués de façon modérce. Le cytoplasme des spermatides allongées est également marqué de façon très modérée. $(\mathrm{pm})=$ noyaux des cellules péritubulaires myoüdes. Barre d'échelle: $10 \mu \mathrm{m}$.

(B) Les noyaux des cellules de Sertoli (S) montrent un marquage plus intense à ces stades. Le cytoplasme des spermatides allongées montre également une réaction plus intense qu'au stade précédent. Une cellule de Leydig ( $L$ ) et des noyaux de cellules péritubulaires myoüdes (pm), positifs pour le marquage des récepteurs aux androgènes, sont visibles. Barre d'échelle: $10 \mathrm{Fm}$.

(C) Avant la spermiation, au stade VIII du cycle de l'épithélium séminifêre, les noyaux des cellules de Sertoli montrent une intensité de marquage maximale. On constate par contre que le marquage pour les récepteurs aux androgènes dans les noyaux des cellules péritubulaires myoüdes $(\mathrm{pm})$, ne varient pàs quant à eux en fonction $d u$ cycle. Les corps résiduels (rb) des spermatozoïdes matures montrent un marquage intense. Au pôle basal de l'épithélium séminifêre on voit la présence de cellules positives mais qui ne peuvent pas être identifiées avec certitude et dont certaines pourraient être des spermatogonies puisque des images similaires sont obtenues lorsque l'on utilise des anticorps contre c-kit. Barre d'échelle: $10 \mu \mathrm{m}$.

la qualité des contrôles effectués. Dans ce but nous avons donc réalisé une série de contrôles conventionnels utilisant PG21 et comprenant à la fois des contrôles positifs et négatifs. Le détail de la méthodologie de ces contrôles à déjà été publié ailleurs [3, 6].

Tout d'abord, nous avons déterminé que PG21 reconnaît bien une bande a $110 \mathrm{kD}$ en Western immunoblot, correspondant au poids moléculaire des récepteurs aux androgènes du rat. Il est clair que la détec- 


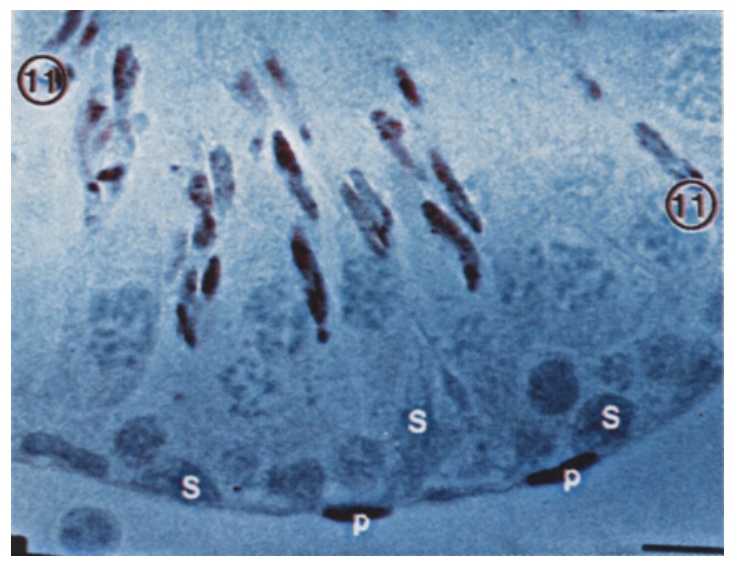

Figure 4. Récepteurs aux androgènes dans les spermatides allongées. Les noyaux des spermatides allongées de stade 11 dont l'élongation est déjà effective mais qui n'ont pas encore commencé leur condensation nucléaire, sont positifs. Dès que le noyau des spermatides allongées commence sa condensation une positivité pour les récepteurs aux androgènes apparaît dans le cytoplasme. Il faut noter qu'à ce stade les noyaux des cellules de Sertoli sont négatifs, mais les cellules péritubulaires myoüdes sont par contre positives.

tion de la bande de $110 \mathrm{kD}$, soit dans les extraits nucléaires, soit dans les extraits cytosoliques de testicules ou de spermatides allongées coïncide très précisément avec les résultats de l'immunocytochimie. En effet les RA sont détectés exclusivement dans la fraction cytosolique dans les spermatides allongées ou les extraits de testicules entiers. Au contraire, l'épididyme et la prostate ne montrent une bande de $110 \mathrm{KD}$ positive que dans les fractions nucléaires ce qui correspond à la localisation décrite sur les coupes. Au cours du développement du testicule et jusqu'à 30 jours après la naissance, les RA sont détectés par immunocytochimie uniquement dans la fraction nucléaire. Là également les résultats par immunoblot coïncident exactement avec la chronologie et la localisation des RA sur coupes. Enfin nous n'avons pas détecté de bande de $110 \mathrm{KD}$ positive dans les testicules de rat âgé de plus de 40 jours. Ces résultats ne sont pas vraiment surprenants puisque les spermatides allongées ainsi que les noyaux des cellules de Sertoli sont déjà très faiblement positifs par immunocytochimie sur coupes. Nous sommes donc en dessous du seuil de détection probable.

Les contrôles cellulaires et tissulaires positifs pour valider la spécificité de l'anticorps anti PG21 ont été réalisés de la façon suivante :

- La partie proximale et distale de la tête de l'épididyme a été traitée par immunocytochimie dans les mêmes conditions que celles utilisées pour le testicule. Sur ces coupes le marquage par les anticorps anti RA se trouve limité au noyau des cellules de l'épithélium de la paroi et au noyau des cellules interstitielles. La localisation du marquage dans ces contrôles positifs est donc exactement celle que l'on attendait en fonction des résultats précédents [5]. De plus, la préadsorption de PG21 avec le peptide antigène élimine tout marquage.

- La lignée de cellules prostatiques LnCAP, connue pour présenter des récepteurs aux androgènes est bien colorée avec les anticorps anti RA. Là également la positivité est très spécifiquement limitée au noyau.

- Le dernier contrôle positif a consisté à transfecter des cellules COS-7 avec le gène des récepteurs des androgènes et à localiser ces récepteurs dans cette lignée cellulaire cultivée soit en présence soit en absence de concentration physiologique d'androgène. Les cellules non transfectées COS-7 restent négatives avec les anticorps anti RA ; après transfection, 10 à $15 \%$ de la population cellulaire se montre positive pour les anticorps anti RA.

De façon très caractéristique, en l'absence d'androgène la distribution des RA est essentiellement cytoplasmique alors qu'après un traitement avec des androgènes, même après des délais aussi brefs que 15 minutes, le marquage se trouve transloqué au niveau du noyau. 
Ces observations sont en accord avec les résultats de Wilson qui a démontré que la répartition nucléocytoplasmique des RA était androgènodépendante [8].

- Enfin, des coupes sériées de testicule ont été soumises à des dilutions croissantes de l'anticorps primaire afin de déterminer si le marquage des spermatides allongées disparaissait à la même dilution que dans les noyaux des cellules de Sertoli ou les cellules myoïdes péritubulaires. Cette étude était donc à même de préciser si le Khd de l'anticorps était le même lorsqu'il interagissait avec des composants nucléaires ou des composants cytoplasmiques. L'étude de l'intensité du marquage des différentes coupes avec ces dilutions montrent que le $\mathrm{Kd}$ est approximativement le même pour les différents sites positifs examinés.

Les contrôles négatifs réalisés pour tester la validité du marquage par PG21 ont été réalisés de la façon suivante :

- PG21 a été préadsorbé avec le peptide antigène ainsi que d'autres peptides des RA de rat, et les anticorps préadsorbés ont été utilisés ensuite pour l'immunocytochimie. La préadsorption de PG21 avec le peptide antigène élimine complètement les réactions positives, alors que les peptides sans réaction avec l'antigène n'ont pas d'effet sur l'intensité du marquage.

- Nous avons refait les mémes études d'immunodétection en utilisant le méme anticorps secondaire mais couplé à un fluorochrome à la place de la peroxidase. En immunodétection par fluorescence nos résultats sont tout à fait superposables à ceux obtenus par anticorps couplé à la péroxidase. Ces résultats montrent en particulier que le marquage des spermatides allongées n'est pas un artéfact puisque nous avons observé la même positivité par immunofluorescence.

\section{DISCUSSION}

Dans ce travail nous montrons par immunocytochimie la distribution des récepteurs aux androgènes dans le testicule de différents rongeurs. La présence de récepteurs aux androgènes dans les cellules somatiques du testicule est en accord d'une part avec certaines études fonctionnelles et des résultats de localisation immunocytochimique (pour revue, 9), d'autre part avec les conjectures théoriques très élégamment formulées par Irving Fritz [1]. De plus, le fait que l'intensité du marquage des RA dans les noyaux de cellules de Sertoli, à la différence de ce que l'on voit au niveau des cellules péritubulaires, varie en fonction du cycle de l'épithélium séminifère pour atteindre un maximum au stade VII-VIII, est en accord avec le fait que ces stades sont les premiers à manifester des altérations histologiques en réponse à la déprivation en androgènes comme cela a été montré par le passé [10].

Ce point avait permis de suggérer que la régulation de la spermatogenèse par les androgènes s'effectue principalement via les cellules de Sertoli. De plus, des observations similaires concernant la distribution stade sécifique des RA chez les rongeurs avaient déjà été faites par d'autres, à la fois par des approches immunocytochimiques [4] et par la mesure relative du taux de mRNA par des techniques d'hybridation in situ [11], les primates faisant cependant exception à cette règle puisque il n'a pas été montré chez eux de distribution similaire des RA en fonction du stade de l'épithélium séminifere [12]. Au total, ces observations fournissent de bons arguments pour dire que la testostérone doit réguler la spermatogenèse. Il faut cependant insister sur le fait que le niveau précis d'action de la testostérone à l'échelle cellulaire reste à élucider. En particulier les évènements transcriptionnels initiés par l'interaction du stéroïde avec son récepteur qui est, selon toute vraisemblance, la base de l'action régulatri- 
ce de la testostérone sur la spermatogenèse, restent à identifier.

La présence d'un marquage positif pour les RA dans les spermatides allongées, cependant, s'écarte du concept généralement admis du mode d'action de la testostérone sur la régulation de la spermatogenèse. En effet, à part quelques résultats ponctuels au cours des 20 dernières années [13-16], la plupart des publications concernant la distribution des récepteurs aux androgènes dans le testicule sont en faveur de l'hypothèse classique formulée par Fritz [1], à savoir qu'aucune cellule de lignée germinale n'est apte à répondre directement aux androgènes.

Dans les résultats présentés ici ainsi que dans les études similaires faites jusqu'à présent $[3,7]$ tous les contrôles effectués concernant l'immunomarquage de RA dans les spermatides allongées confirment que ces récepteurs doivent bien y étre présents, en particulier dans les noyaux des spermatides de stade XI et dans le cytoplasme des spermatides plus différenciées. Nos résultats conduisent donc à conclure que certaines cellules de la lignée germinale présentent des récepteurs aux androgènes et sont donc susceptibles de répondre directement à la testostérone. Bien sûr, en l'absence d'étude fonctionnelle, il ne nous est pas possible d'affirmer que la présence de ces récepteurs intervient directement dans la réponse de la lignée germinale aux androgènes. Néanmoins nos résultats conduisent, comme d'autres, à évoquer la possibilité d'une telle régulation directe en plus de celle qui est déjà connue via des cellules somatiques. Il est intéressant de noter que quelques expérimentations suggèrent la possibilité d'une réponse des cellules germinales à l'action directe des androgènes, probablement médiées par un récepteur aux androgènes. Tout d'abord, les spermatides rondes isolées et purifiées synthétisent et secrètent deux protéines spécifiques en réponse au traitement par les androgènes [18].
Deuxièmement l'addition d'ABP et de testostérone sur les cellules germinales isolées diminue de façon significative l'incorporation de thymidine tritiée par rapport à l'adjonction d'ABP seule [18].Une explication de ces résultats serait que les lignées germinales répondent directement à la fois à l'ABP et à la testostérone. Troisièmement, la distribution des RA dans les spermatides allongées est surperposable à celle de l'aromatase [19], une autre protéine qui lie les androgènes. Au total, ces résultats fonctionnels et nos propres résultats, conduisent à poser la question de savoir pourquoi certaines équipes rapportent la détection de RA dans les cellules germinales $[3,7,15$, 16], alors que d'autres font état de leur absence $[2,4,12,20]$ ? La réponse la plus logique à cette question est premièrement que l'immunocytochimie est à la fois une technique particulièrement puissante pour montrer la distribution d'une protéine dans les tissus, mais que en méme temps elle n'est pas une méthode qui permet de démontrer qu'une protéine ne s'y trouve pas. Par ailleurs l'utilisation de différents types d'anticorps, de différents types de colorations, de fixations ainsi que l'utilisation de différentes espèces animales contribue à fournir une vision très disparate des résultats obtenus par différentes équipes. Il convient donc de poursuivre ces recherches.

La démonstration récente que les spermatogonies de souris présentent une réaction positive avec les anticorps anti RA mérite également discussion [21]. Bien que nous n'ayons pas pu obtenir la preuve définitive que certains noyaux positifs pour les anticorps anti RA localisés à la base de l'épithélium du tube séminifere soient des spermatogonies, nos résultats ont montré que certaines images étaient très superposables aux colorations des tubes séminifêres par c-Kit, un marqueur spécifique des spermatogonies. Ces observations nous conduisent à conclure que certaines cellules RA positives doivent être des spermatogonies ce 
qui corrobore les découvertes récentes de Hirano et collaborateurs [21]. Il est intéressant de noter que ces résultats concordent avec deux études antérieures décrivant la présence de RA dans les spermatogonies humaines et leur implication possible dans les cas d'infertilité masculine et/ou comme élément prédisposant à l'apparition de séminomes [15-16].

\section{REFERENCES}

1. FRITZ I. : Sites of action of androgens and follicle stimulating horrnone on cells of the seminiferous tubule. In : G Litwack edit., Biochemical Actions of Hormones, Academic Press, New York 1978 ; 249-281.

2. SAR M., LUBAHN D.B. FRENCH F.S., WILSON E.M. : Immunohistochemical iocalization of the androgen receptor in rat and human tissues. Endocrinology, $1990 ; 127,3180-3186$.

3. VORNBERGER W., PRINS G., MUSTO N.A., SUAREZ-QUIAN C.A. : Androgen receptor distribution in rat testis : new implications for androgen regulation of spermatogenesis. Endocrinology, 1994 ; 134, 2307-2316.

4. BREMNER W.J., MILLAR M.R., SHARPE R.M., SAUNDERS P.T.K. : Immunohistochemical localization of androgen receptors in the rat testis : evidence for stage-dependent expression and regulation by androgens. Endocrinology, $1994 ; 135$, 1227-1234.

5. PRINS G.S., BIRCH L., GREENE G.L. : Androgen receptor localization in different cell types of the adult rat prostate. Endocrinology, $1991 ; 129$, 3187-3199.

6. OKE B., SUAREZ-QUIAN C.A. : Localization of secretory, membrane-associated and cytoskeletal proteins in rat testis using an improved immunocytochemical protocol that employs polyester wax. Biol. Reprod., 1993 ; 48, 621-631.

7. SUAREZ-QUIAN C.A., OKE B., VORNBERGER W., PRINS G., XIAO S., MUSTO N.A. Androgen receptor distribution in the testis. In : C Desjardins. edit., Cellular and Molecular Regulation of Testicular Cells, Norwell, MA: Serono Symposia USA 1996 ; 189-212.

8. SIMENTAL J.A., SAR M., LANE M.V., FRENCH F.S., WILSON E.M. : Transcriptional activation and nuclear targeting signals of the human androgen receptor. J. Biol. Chem., $1991 ; 266$,
510-518.

9. SHARPE R. : Regulation of spermatogenesis. In : E Knobil, JD Neill, eds, The Physiology of Reproduction, second edition, Raven Press, New York 1994; 1363-1434.

10. RUSSELL L.D., CLERMONT Y. Degeneration of germ cells in normal, hypophysectomized and hormone treated hypophysectomized rats. Anat. Rec., $1977 ; 187,347-366$.

11. SHAN L., ZHU L., BARDIN C.W., HARDY M.P. : Quantitative analysis of androgen receptor messenger ribonucleic acid in developing Leydig cells and Sertoli cells by in situ hybridization. Endocrinology, 1995, 136, 3856-3862.

12. SAUNDERS P.T.K. MILLAR M.R., MAJDIC G., BREMNER W.T., McLAREN T.T., GRIGOR K.M., SHARPE R.M. : Testicular androgen receptor protein : distribution and control of expression. In : C Desjardins, edit., Cellular and Molecular Regulation of Testicular Cells, Norwell, MA : Serono Symposia USA 1996, 213-229.

13. WRIGHT W.W., FRANKEL A.I. : An androgen receptor in the nuclei of late spermatids in testes of male rats. Endocrinology, 1980, 107, 314- 318.

14. SCHULZ R., PARIS F., LEMBKE P., BLUM V. : Testosterone immunoreactivity in the seminiferous epithelium of rat testis : effect of treatment with ethane demethanesulfonate. J. Histochem. Cytochem., 1989, 37, 1667-1673.

15. RAJPERT-DE MEYTS E., SKAKKEBAEK N.E. : Immunohistochemical identification of androgen receptors in germ cells neoplasia. J. Endocrinol., 1992, l 35, R I -R4.

16. KIMURA N., MIZOKAMI A., OONUMA T., SASANO H., NAGURA H. : Immunocytochemical localization of androgen receptor with polyclonal antibody in paraffin-embedded human tissues. J. Histochem. Cytochem., 1993, 41, 671678.

17. McKINNELL C., SHARPE R.M. : Testosterone and spermatogenesis : evidence that androgens regulate cellular secretory mechanisms in stage VI-VIII seminiferous tubules from adult rats. J. Androl., 1995, 16, 499-509.

18. GERARD A., BEDJOU R., CLERC A., MAACHI F., CLOSSET J., HAMMOND G.L., NABET F., GERARD H. : Growth response of adult germ cells to rat androgen-binding protein and human sex hormone-binding globulin. Hor. Res., 1996, 45, 218-221.

19. NITTA H., BUNICK D., HESS R.A., JANULIS L., NEWTON S.C., MILLETTE C.F., OSAWA Y., SHI- 
ZUTA Y., TODA K., NAHR J.M. : Germ cells of the mouse testis express P450 aromatase. Endocrinology, 1993, 132, 1396-1401.

20. GOYAL H.O., BARTOL F.F., WILEY A.A., NEFF C.W. : Immunolocalization of receptors for androgen and estrogen in male caprine reproductive tissues : unique distribution of estogen receptors in efferent ductule epithelium. Biol. Reprod., 1997, $56,90-101$.

21. ZHOU X., KUDO A., KAWAKAMI H., HIRANO $H$. : Immunohistochemical localization of androgen receptor in mouse testicular germ cells during fetal and postnatal development. Anat. Rec., 1996, 245, 509-518.

\section{ABSTRACT \\ Distribution of the androgen receptor in the testicle \\ C.A. Suarez-Quian, B.O. OKe, W. WorN- BERGER}

Whether or not germ cells contain the androgen receptor remains a matter of controversy. In the present study we performed biotinstreptavidin immunoperoxidase using an affinity purified rabbit polyclonal antibody made to a 21 amino acid peptide of the amino terminus of the rat $A R$ to determine androgen receptor (AR) distribution in the rat and mouse testes. Specificity of the antibody was confirmed as follows : 1) Western immunoblots rendered a specific band at approximately $110 \mathrm{kD} ; 2)$ preadsorption of the antibody with the 21 amino acid peptide eliminated specific immunostaining ; 3) the intensity of staining in all AR positive cells diminished as a function of antisera dilution ; 4) tissues known to express abundant AR (e.g.,epididymis, prostate, seminal vesicles) all rendered a robust, nuclear AR immunostaining pattern in the epithelial cells ; 5) prostate cell lines known to express
AR immunostained positive with the antibody ; 6) AR negative COS-7 cells became AR immunopositive when transfected with a vector expressing the rat $A R$ and intracellular $A R$ distribution was a function of androgens. AR immunostaining results revealed the following : Within the interstitial compartment of adult rats, AR was detected in some Leydig cells and all smooth muscle cells forming the walls of blood vessels, but endothelial cells were negative. In the seminiferous tubules AR was observed in all peritubular myoid cell nuelei, but not in the distal layer of Iymphatic endothelial cells. In Sertoli cells, nuclear AR immunostaining was stage specific; moderate AR immunostaining became evident at late stage IV of the cycle, reached a robust peak at stages VII-VIII, and then disappeared completely. Specific AR immunostaining was also discerned in the nuclei of stage XI elongated spermatids, in which nuclear elongation is apparent but chromatin condensation has not yet begun. With onset of chromatin condensation, nuclear AR immunostaining in elongated spermatids was not discerned concomitant with its detection in the cytoplasm. In general, similar observations have now been confirmed in the adult mouse testis, except that an Leydig cells were strongly AR positive. Nucleic acid in situ hybridization studies for $A R$ were performed in adult rat testis using a 236 bp antisense cRNA probe (rat AR cDNA was provided by Dr. C. Chang, U. Wisconsin, Madison, WI) to confirm the AR immunostaining. A prominent hybridization signal at the base of the seminiferous epithelium was observed, in the 
area occupied by Sertofi and spermatogonia. This led us to re-examine the immunostaining results to determine if spermatogonia were also AR positive. Preliminary results are consistent with the interpretation that AR is present in certain spermatogonial populations. Taken together, these results concur with prior observations suggesting that $A R$ is present in the somatic cells of the testis; thus, it is these cell types that likely respond to circulating androgens to control spermatogenesis. However, they raise anew the controversy of whether germ cells respond directly to androgens.

Key Words : testis, germ cells, androgen receptor-androgen, immunohistochemistry. 